\title{
EYE AND HEAD MOVEMENTS IN THE ACQUISITION OF VISUAL INFORMATION
}

\author{
John A. STERN'), Timothy B. BROWN', Lin WANG ${ }^{2)}$ \\ ${ }^{1)}$ Washington University, U.S.A., ${ }^{2)}$ University of Salzburg, Austria \\ and \\ Michael B. RUSSO ${ }^{3)}$ \\ ${ }^{3)}$ Walter Reed Army Institute of Research, U.S.A.
}

\begin{abstract}
Much of the literature dealing with the measurement of eye movements is restricted to data collected under conditions where the head is "stabilized". Since in the "real world" such restrictions cannot readily be imposed on operators, we here review the literature dealing with the use of head movements in the acquisition and processing of visually presented information. Task difficulty is associated with an increase in the likelihood of using head movements to acquire visually presented information. Such head movements have been described with visual information subtending as little as 5-10 degrees of arc.

The present study, requiring subjects to identify and process information presented at 10 degree eccentricity, determined that such head movements do occur. Head movements were not measured directly but inferred from differences in eye movement amplitude as measured with electrooculography, and gaze shift measured with video camera technology. We raise the issue of generalizability of results obtained under conditions where the head is "stabilized" to conditions where it is free to move.
\end{abstract}

Key words: gaze, eye movement, EOG, head movement, saccade

Much of the literature dealing with the evaluation of saccadic (and other) eye movements utilizes experimental paradigms where the head is stabilized by a bitebar, and/ or chin and forehead rest. The study of oculomotor measures in real world situations requires a reduction of such constraints. For example, military members are using Helmet-Mounted Displays (HMDs, also referred to as Heads up Displays or HUDs) that stream digitized visual information to a transparent lens or visor with the image projected and focused at infinity (Rash et al., 1998). Some HMDs present information to a single eye, while others present to both eyes simultaneously (McLean \& Estrada, 2000). In addition to attending to information projected onto the Helmet-Mounted Display, the

Funding was provided by the Saccadic Fatigue Measurement Research Program, U.S. Army Medical Research and Materiel Command, Ft. Detrick, Maryland.

We thank our volunteers for their participation, and the students who helped in analyzing data. We thank Dr. William McLean and LTC Corina Van de Pol from the U.S. Army Aeromedical Research Laboratory, Fort Rucker, Alabama, for reviewing this manuscript and providing substantive insight into the relevance of this research to the aviation community.

Correspondence concerning this article should be addressed to John A. Stern, Department of Psychology, Box 1125, Washington University, One Brookings Drive, St. Louis, MO 63130, U.S.A. (e-mail: jastern@ artsci.wustl.edu). 
operator must also attend to the external visual environment. Visual display systems are also frequently yoked to gaze location. As the technologies that provide information advance and the information streams become both more dense and more rapid, the challenge both to oculomotor physiology and to visual cognition ability steadily increases.

What effect does removing the restriction on head movement have on eye movements? As may be obvious, from the fact that saccadic eye movements are generally measured with the head stabilized, there must be significant effects on the measurement of such eye movements when that constraint is lifted.

\section{Background}

How important are head movements in the acquisition of visual information? There is a considerable literature demonstrating that with stimulus eccentricities of 20 degrees or more some portion of the gaze shift may be accomplished by moving the head (Goldring, Dorris, Corneil, Ballantyne, \& Munoz, 1996). This is especially true when it takes more than a fraction of a second to acquire the peripherally presented information. Robinson, Koth, and Ringenbach (1976) for example demonstrated that when subjects were required to search for a specific digit in a matrix of digits (4 or 8), at target eccentricities of 60, 80, and 100 degrees, head movements occurred $100 \%$ of the time; at 40 degrees, approximately $75 \%$ of the time; and at eccentricities of less than 20 degrees, less than $2 \%$ of the time. The likelihood of head movement was affected by stimulus brightness but not by task difficulty. They also noted, as an aside, that the use of a calibration procedure (bite bar) prior to task performance inhibited head movements during task performance. Bartz (1966) reported no head movements for stimuli with eccentricity less than 40 degrees for subjects performing a simple stimulus identification task. Fuller (1992), based on studies using targets at 20 and 40 degree eccentricities identified subjects as "head movers" and "non head movers" and came to the conclusion that, "Individuals have an innate propensity to move their heads ..." p 156. Zangemeister, Jones, and Stark (1981) came to the conclusion that: "In addition, the head movement depends a good deal on subject intent; this, in turn, is influenced by his skill and experience and the nature of his perception of instructions given during the experiment." $\mathrm{p}$ 86. They further suggest that fatigue and "central nervous system habituation" may affect likelihood of head movement occurrence.

Sanders (1970), using a simple discrimination task, suggested that the "stationary field", where stimuli can be apprehended without eye movements, extends to 20-30 degrees into the periphery. The "eye field', where the eyes have to move in order to acquire information, extended out to 75 degrees, while the "head field" is limited to visual angles greater than 75 degrees. Sanders suggested that these boundaries are rather flexible, varying with perceptual difficulty. Increasing perceptual complexity decreases the boundaries of the visual field. Van de Pol, a military research optometrist specializing in the study of eye movements of Army Aviators, describes pilot's visual field in similar terms, with the field of vision encompassed by the typical night vision scope subtending an arc of up to 40 degrees, while all vision beyond this is referred to as the field of visual regard and viewed with head movements (unpublished communication 2003). Pilots, 
especially where there is a limited field of view due to the restrictions inherent in using night vision scopes, repeat a pattern of eye and head movements intended to direct attention to all regions of the field of regard. This repeated pattern becomes an almost unconscious activity in experienced and highly skilled pilots (Stern \& Bynum, 1970). The use of head movements during visual information acquisition is most reliably demonstrated in young children. Richards and Hunter (1997), using 14, 20, and 26 week old infants and stimulus eccentricities of 25,35 , and 45 degrees reported that localization of the object was accompanied by head movements on more than two-thirds of the trials, with likelihood of head movement positively associated with eccentricity. They quote Tomlinson and Bahra (1986a, 1986b) as indicating that saccades occurring during head movements are slower than those occurring under head stabilized conditions.

Again, one can readily demonstrate that such head movements are more prevalent in infants than in adults. Observe an infant in the mother's lap, with both of them looking at objects presented in front of them at eccentricities of 10-20 degrees. The infant will use head and eye movements to acquire the information while the mother is most likely to use only her eyes. Watching children in front of a television set should also be instructive in demonstrating that the younger the child the greater the likelihood of using a combination of head and eye movements to view the display.

Are these eye/head movement boundaries affected only by perceptual complexity or can cognitive complexity affect them similarly? Here we have a considerably smaller research base on which to draw. Dunham (1997) studied the effect of manipulating cognitive difficulty with stimuli presented at a 40-degree target eccentricity. He demonstrated that increasing cognitive difficulty significantly increased the likelihood of making head movements. Approximately $30 \%$ of his subjects made no head movements during either the simple or more difficult task conditions. These subjects also made significantly fewer errors in performing the arithmetic task, suggesting that the task was easier for them than for subjects who made head movements. However, response latency, another measure reflecting task difficulty, did not discriminate between the groups. Dunham suggested that small sample size $(N=24)$ may have accounted for the lack of discriminability of the reaction time measure.

Most recently Lee (1999), using text subtending a rather large visual angle (50 or 90 degrees) reported that “... head movements are strongly influenced by cognitive factors, and therefore in head-free reading they may predominantly reflect the cognitive influences that have been shown for eye movements in head-fixed reading, and thereby decouple eye-head coordination." p 3761. He found the pattern of head movement during reading to be complex. The fact that he used unusually long line lengths in this study somewhat reduces its impact. In a personal communication (2003) he indicated that he had recorded head movements during the reading of "normal" text, i.e., 6-10 degree line length, and found such head movements, but to a much smaller extent than with 50 degree line length.

Earlier studies utilized text with line lengths closer to "normal" line length. Fischer (1924) reported that during reading one could almost always observe head movements. The head movement returning the eyes from the end of a line to the beginning of a new line is readily apparent, while the right-going movements are "slow and regular and less 
easily observed." He further suggested that such movements are most apparent in children and "unpracticed" readers. He systematically studied students between the ages of 11 and 20, manipulating line length to elicit head movements. He found that 11 year olds were likely to use head movements with $6 \mathrm{~cm}$ line length while 17-20 year olds only showed head movements when line length was $8-10 \mathrm{~cm}$ long. Reading aloud produced head movements with $6 \mathrm{~cm}$ lines in all subjects. According to Fischer, studies by Erdman and Dodge indicated that unpracticed readers are more likely to make upper body and head movements than is true of practiced readers. Fischer also reviewed earlier work by Ritzman (1875) and Hering (1879). Ritzman had readers clench a pointer between the teeth and observed movements of the pointer while subjects read. He reported that even with the smallest gaze change one can observe head movements and that there were marked individual differences in the likelihood of such head movements. Hering used himself as the object of study. He reported that if one read with a cigar or pencil in the mouth, one can readily observe gaze changes accompanied by head movements during reading. If the head moves, location of the tip of the cigar with respect to the text changes. These findings are directly relevant to aviators learning to fly with the use of night vision scopes and HMDs in that the opposite occurs. Novice aviators display a paucity of head movements as they appear to focus on assimilating the complex visual information displayed within the cockpit while more skilled aviators demonstrate an increase in head and eye movements as they develop the repetitive scanning pattern intended to integrate the entire visual field of regard (Troy, Chen, \& Stern, 1972).

More recently, Netchine, Greenbaum, and Guihou (1984), Netchine, Pugh, and Guihou (1987) and Netchine, Solomon, and Guihou (1981) studied head movements in children and adults as they read silently and aloud. They report that for both groups, head movements are more likely to occur under reading aloud than reading silently conditions. They also manipulated conceptual text difficulty by requiring readers to read text in both their "native" language and a "foreign" language with which the readers were familiar. Reading a foreign language elicited more head movements than reading in one's native language. Younger children were reported as more likely to utilize head movements than older children. The text used by these authors was usually $27 \mathrm{~cm}$ long, again a length considerably longer than found in "normal" printed text.

We have observed head movements during reading text subtending no more than 10 degrees of visual angle in many readers. The observed head movement is usually restricted to shifting gaze from the end of a line to the beginning of a new line. It is a phenomenon that one can readily demonstrate. Ask a reader to read aloud from text where the line length is between (the usual) 6-10 degrees. Without looking at the reader's eyes (stand behind the reader) look for head movements. They are small in amplitude but readily observable. You can then tell the reader that you will identify when he shifts gaze from the end of a line to the next line. They will be surprised at the accuracy with which you can make that prediction, and until you tell them that it is based on the fact that they move their head, they will not be aware of the head movement. You will not be able to observe the head movement with every line change, but with many, especially if the text is conceptually difficult for the reader. Should you have the opportunity to visit a college or 
high school classroom where remedial reading is being taught you will see that most of the students, even when reading silently, engage in head movements, and in some cases mouth movements as well. With regard to the acquisition of increasingly complex visual information in HMDs, will head movements not only reflect increasing task difficulty during the attempts to capture challenging visual information, but might also these unconscious head movements more quickly fatigue military operators with yoked displays and the extra weight of helmets? Might the frequency and amplitude of head movements increase with impending "overload"?

\section{EXPECTATIONS}

If head movements are "normally" used in shifting gaze, what effect does restricting head movements have on our ability to make inferences about gaze shifts in the acquisition of visual information? Conversely, how does lifting this restriction affect our ability to measure gaze shift inferred from the recording of eye movements alone? We will deal with the latter, not the former question by exploring the relationship between saccade amplitude as recorded with electrooculography (EOG) and camera technology (Camera) as well as the relationship between saccadic eye movements recorded with EOG under the conditions of "calibration" and "task performance." What might we expect?

\section{Relationship between saccade amplitude as recorded with EOG and Camera technology}

Data acquired using Camera technology captures gaze, rather than eye position. Gaze position can be changed by eye movements, head movements, or a combination of the two. Thus saccade amplitude, as measured electrooculographically, and gaze shift amplitude should, under conditions where there is no head movement, correlate highly. Under conditions where head movements occur some of the time, the correlation should be considerably lower.

Relationship between saccade amplitude and duration measured with EOG under the conditions of "calibration" and "task performance"

If, under head-restricted conditions, there is a high correlation between saccade amplitude and saccade duration (Baloh, Sills, Kumley, \& Honrubia, 1975) one would expect, on the basis of the study by Tomlinson and Bahra (1986a, 1986b), that under nonrestricted-head conditions, saccades may become slower when the head moves and therefore the correlation would be lower because we are dealing with a mixture of saccades with and without head movements.

\section{METHODS}

Subjects:

Eight undergraduate students (4 male and 4 female subjects, mean age 20, range 19-23) volunteered to participate in this study for course credit. 
Procedure:

Subjects came to the laboratory and were instrumented for the recording of horizontal and vertical EOG. DC amplifiers with automatic reset capability and cutoff at $100 \mathrm{~Hz}$ and a $60 \mathrm{~Hz}$ notch filter were used. They then went into the recording chamber and equipment was adjusted for recording eye activities using pupil-center/corneal-reflection technology (referred to as Camera in the following text) (L.C. Technologies, Inc., Eyegaze System). Subjects sat in a military-style dental chair with a headrest adjusted for maximal comfort for the subject. The headrest contacted the back of the head at two points and allowed for head movements. Calibration data involving three locations to the left and right of center (3, 6, and 9 degree eccentricities) was collected over approximately one minute. Four samples were collected at each of the six locations. This procedure was performed prior to onset and again at the completion of the 60-minute experimental period.

The experimental task required subjects to move their eyes from a central location to target locations 10 degrees to the left or right of center. Target location was not predictable. Numerals were presented at the peripheral locations and subjects were required to make a simple manual response when they had seen a sequence of two odd integers. Stimulus duration was $400 \mathrm{~ms}$ with an interstimulus interval of $2000 \mathrm{~ms}$. Over a 60 -minute period this allowed for the presentation of 1500 stimuli, of which 90 involved the presentation of a sequence of two odd integers and 30 involved the presentation of a single odd integer. Numerals were flanked by the letter $\mathrm{X}$ in a stimulus presentation $(\mathrm{X} \# \mathrm{X})$. At the termination of a stimulus, the central stimulus was presented consisting of an array of three X's (XXX). The onset of a peripheral stimulus terminated the central stimulus.

Data was digitized at $1000 \mathrm{~Hz}$ and stored on the hard drive of a PC. The abstracted data included stimulus and response information, EOG (2 channels - horizontal and vertical eye movements) and Camera (3 channels- horizontal and vertical gaze positions and pupil diameter) data. Since the Camera data was actually produced at $60 \mathrm{~Hz}, 16$ consecutive samples of the Camera data were identical in the $1000 \mathrm{~Hz}$ recording. Data abstraction utilized a procedure which automatically identified saccades in the horizontal plane, eye blinks in the vertical EOG, as well as stimulus information (onset, termination, 1st odd, 2nd odd, even integer) and response information. Data was visually scanned to identify missed or incorrectly identified stimuli, responses, saccades and blinks, and edited. Edited events were identified as such in the output files.

Saccades occurring during a blink were excluded from consideration since McGregor and Stern (1996) had demonstrated that many such saccades were slower than those occurring independent of blinks.

\section{RESULTS}

\section{Comparison of EOG and Camera Saccade Amplitude Data}

EOG saccade amplitude reflects eye position shifts with respect to the head, while Camera saccade amplitude reflects gaze shift with respect to the display. A gaze shift can be accomplished by eye movements alone or a combination of eye and head movements. If gaze shift is accomplished by eye movements alone, then the correlation between saccade amplitude as measured with the two procedures (EOG and Camera) should be high. If, on the other hand, the gaze shift is accomplished by a combination of head and eye movements then the correlation should be lower.

Saccades identified as "to-Target" (tT) and "return-from-Target" (rT) were first evaluated in the EOG-based data. This included data associated with calibration runs before and after each experiment as well as data associated with the experiment. Saccade identification was performed using computer software, PCWUPDRS, which was developed in our laboratory and is available from the first author (JAS). The identified saccades were further edited by visual inspection if necessary. Only saccades meeting the criterion of being associated with the eyes moving to and returning from a target location 
Table 1. Pearson Product-Moment Correlations for Saccades Identified as "to-Target" (tT) and "returnfrom-Target" (rT) Saccades

\begin{tabular}{|c|c|c|c|c|c|c|}
\hline \multirow{2}{*}{ Subject } & \multicolumn{2}{|c|}{ Initial cal. } & \multicolumn{2}{|c|}{ Final cal. } & \multicolumn{2}{|c|}{ Task perf. } \\
\hline & $\mathrm{tT}$ & rT & $\mathrm{tT}$ & rT & $\mathrm{tT}$ & $\mathrm{rT}$ \\
\hline F41 & .98 & .98 & .94 & .96 & .67 & .62 \\
\hline F42 & .98 & .99 & .99 & .99 & .85 & .68 \\
\hline F43 & .91 & .99 & .99 & .99 & .87 & .91 \\
\hline F44 & .96 & .98 & .96 & .98 & .67 & .65 \\
\hline M41 & .98 & .98 & .99 & .97 & .71 & .85 \\
\hline M42 & - & - & .99 & .99 & .48 & .40 \\
\hline M43 & .98 & .99 & .95 & .96 & .71 & .66 \\
\hline M44 & .98 & .97 & .96 & .96 & .69 & .41 \\
\hline Average & .97 & .98 & .97 & .98 & .72 & .67 \\
\hline
\end{tabular}

were evaluated. To measure saccade amplitude in the Camera data we sampled that data $20 \mathrm{~ms}$ before and $50 \mathrm{~ms}$ following termination of the EOG-identified saccade. The results are depicted in Table 1.

It is readily apparent that the correlations between EOG saccade amplitude and Camera saccade amplitude are robust for all correlations involving the calibration data. All these correlations are greater than 0.90 (Mean=.974, $S D=.019)$. There are no differences between the pre-task as compared to post-task calibration runs nor between $\mathrm{tT}$ and rT saccades. Correlations between the two measures during task performance are considerably lower and none of them overlap with the calibration-based correlations. (Mean=.667, $S D=.214$, excluding data for subject $\mathrm{M} 42$ ). There is considerable variation in the level of correlation across subjects suggesting that some of the subjects moved their heads more frequently than others during task performance.

For task-related saccades the analysis is based on many more tT saccade events than rT saccade events since we excluded from consideration any saccade that occurred during a blink. Return saccades are more likely to have concurrent eye blinks, again confirming our hypothesis that blinks occur at points in time where information acquisition and processing is minimal. We had earlier demonstrated that such saccades are related to blink duration (McGregor \& Stern, 1996).

To more formally test the idea that return saccades are more likely to occur concurrent with a blink, we calculated the ratio of the number of rT to tT saccades for successive five minute periods for stimuli not requiring a response. All saccades that occurred concurrent with an eyeblink had been removed from the database. Thus, if there were more saccades associated with a blink for $\mathrm{rT}$ than $\mathrm{tT}$ we should have fewer $\mathrm{rT}$ saccades evaluated. For any five-minute period there were approximately 100 stimuli 


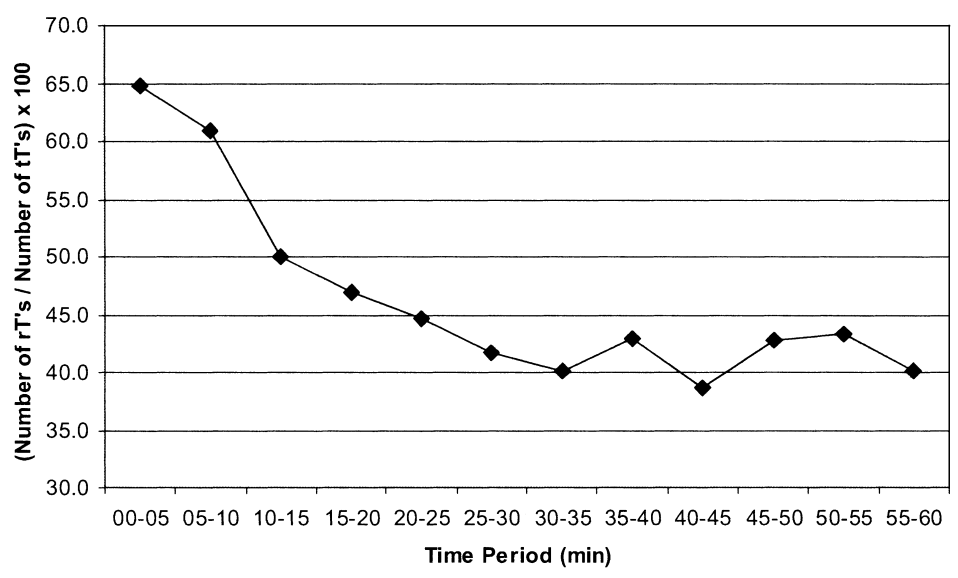

Fig. 1. Ratio of rT to tT saccades. $(\times 100)$

Table 2. Number of Saccades Evaluated for "to-Target" and "return-from-Target" Saccades

\begin{tabular}{|c|c|c|c|c|c|c|}
\hline \multirow{2}{*}{ Subject } & \multicolumn{3}{|c|}{$\mathrm{tT}$} & \multicolumn{3}{|c|}{$\mathrm{rT}$} \\
\hline & $60 \mathrm{~min}$ & Mean/5 min & $S D$ & $60 \mathrm{~min}$ & Mean $/ 5$ min & $S D$ \\
\hline F41 & 1105 & 92.1 & 3.45 & 610 & 50.8 & 18.68 \\
\hline $\mathrm{F} 42$ & 1126 & 93.8 & 2.41 & 1050 & 87.5 & 7.20 \\
\hline F43 & 1063 & 88.6 & 5.07 & 220 & 18.3 & 6.00 \\
\hline F44 & 1156 & 96.3 & 2.96 & 337 & 28.1 & 7.97 \\
\hline M41 & 1150 & 95.8 & 3.33 & 628 & 52.3 & 22.14 \\
\hline M42 & 1144 & 95.3 & 1.50 & 557 & 46.4 & 14.16 \\
\hline M43 & 1130 & 94.2 & 4.26 & 365 & 30.4 & 24.31 \\
\hline M44 & 1096 & 91.3 & 2.99 & 400 & 33.3 & 13.06 \\
\hline
\end{tabular}

requiring no responses. As depicted in Fig. 1 the number of return saccades for even the first five minutes of task performance was approximately $65 \%$ of $\mathrm{tT}$ saccades during this same period. There was a marked decrease in this ratio, asymptoting at about $40 \%$ after 25 minutes of task performance. Changes in ratios can occur as a function of either or both the numerator and denominator changing in value. To demonstrate that the effect was principally attributable to $\mathrm{rT}$, the numerator in the equation, we calculated the mean and standard deviation for each subject over the 12 five-minute periods of the experiment. Table 2 depicts the results of this analysis. It is readily apparent that the effect is principally attributable to changes over time for the rT saccades. Not only are the number of saccades evaluated for $\mathrm{rT}$ consistently lower than those for tT but variability over time, as reflected in the $S D$ for data sampled at 5-minute intervals are consistently greater for the rT saccade measure. 
Table 3. Correlation Between EOG Measured Saccade Amplitude and Saccade Duration

\begin{tabular}{ccccccccc}
\hline \multirow{2}{*}{$\begin{array}{c}\text { Time } \\
(\mathrm{min})\end{array}$} & \multicolumn{7}{c}{ Subject } \\
\cline { 2 - 9 } & $\mathrm{F} 41$ & $\mathrm{~F} 42$ & $\mathrm{~F} 43$ & $\mathrm{~F} 44$ & $\mathrm{M} 41$ & $\mathrm{M} 42$ & $\mathrm{M} 43$ & $\mathrm{M} 44$ \\
\hline $1-10$ & .58 & .92 & .84 & .80 & .76 & .80 & .46 & .60 \\
$11-20$ & .62 & .83 & .79 & .80 & .71 & .85 & .42 & .63 \\
$21-30$ & .59 & .76 & .88 & .85 & .65 & .83 & .37 & .55 \\
$31-40$ & .55 & .82 & .83 & .73 & .67 & .85 & .53 & .59 \\
$41-50$ & .60 & .84 & .88 & .78 & .68 & .80 & .55 & .46 \\
$51-60$ & .55 & .83 & .83 & .77 & .80 & .85 & .50 & .53 \\
Average & .58 & .84 & .84 & .79 & .71 & .83 & .48 & .56 \\
Cal 1 & .91 & .88 & .88 & .85 & .93 & .87 & .87 & .86 \\
Cal 2 & .84 & .94 & .93 & .91 & .90 & .94 & .89 & .86 \\
\hline
\end{tabular}

\section{Saccade Amplitude - Duration Correlation Analysis}

In the comparison of saccade amplitude measured with EOG and Camera, we found lower correlation during task performance than calibration runs, which suggests that head movements were involved in gaze shift during the task performance. In this analysis, we further investigate saccade velocity change, presumably slower when accompanied by head movements. If eyes move at a different velocity during head movement than with the head stabilized, one should see lower correlation between saccade amplitude and duration during task performance where gaze shifts are accomplished by a mixture of eye and head movements than during calibration runs where head movements are less likely to occur.

Saccade amplitude and duration were measured for consecutive 10-minute periods. All saccades were included except those that occurred during an eye blink. Calibration data was collected over a one-minute period, sampling 3 levels of eye-position change to the left and right of the central location. This data set included eye movements to the peripheral location as well as saccades returning the eyes to the central location. Table 3 displays these correlations. Correlations involving task performance are based on a minimum of 300 pairs of measures with the vast majority involving more than 500 pairs. Correlations involving calibration data are based on a minimum of 40 pairs of measures. Because of relatively large sample size all correlations are significantly different from chance (at the $p<.01$ level).

It is readily apparent that the correlations for the calibration conditions are consistently higher than those obtained during task performance, and that there are no systematic changes in the correlations as a function of time on task. There is no overlap between the average correlation during task performance and either of the two calibration runs.

What might account for the dramatic differences in correlation between the 
calibration and task performance condition? We say dramatic differences since there is little overlap between these two sets of values for each individual. Using the lowest correlation value for the calibration condition we find no overlap between this correlation and the 6 measures obtained during task performance for 5 subjects (F41, M41, M42, M43, M44), one value overlapping for 2 subjects (F42, F44) and one subject (F43) with 2 of the 6 correlations during task performance overlapping with the lowest correlation during calibration. It is most likely that head movements were a contributor to the lower correlations between saccade amplitude and duration during task performance as compared to the calibration condition.

In agreement with the findings of Fuller (1992) and Dunham (1997) who reported that subjects can be divided into those who are likely and those who are unlikely to use head movements, we find that the correlations under task performance conditions can be grouped into two sets, those that are most deviant from the calibration conditions (F41, M43, M44) and those that approach the correlations obtained for the calibration condition. The most deviant ones are the ones most likely to be using a combination of head and eye movements during task performance. There does not appear to be a change as a function of time on task, those who used head movements early in task performance continued to do so for the 60-minute performance period and those who demonstrated high correlations also demonstrated no consistent pattern of change over the 60 -minute period.

\section{DISCUSSION}

Our results, as well as our review of the literature, strongly suggest that the likelihood of head movements associated with gaze shifts is not limited to large amplitude gaze shifts, i.e., shifts greater than 20 or 40 degrees. In our study, saccade amplitude seldom exceeded 10 degrees, yet the use of head movements associated with task performance could be readily inferred from the results. The literature dealing with head movements associated with small amplitude saccades is generally limited to studies involving the reading of text. As pointed out in our introduction, this phenomenon was noted as early as 1875 by Hering and formally investigated in 1924 by Fischer and again in the 1980's by Netchine and coworkers and in a study by Lee in 1999. One of us (JAS) has studied it informally for the past 20 years and found it to be a robust phenomenon.

We demonstrate, though indirectly, that eye movements may become slower during head movements. Richards and Hunter (1997) reported an observation by Tomlinson and Bahra (1986a, 1986b) that saccades occurring during head movements are slower than those occurring under head stabilized conditions. Our results support this hypothesis. It further suggests a possible approach of measuring head movements using EOG alone. One would thus raise the question how the eye and head are coordinated during gaze shift and why such coordination slows down eye movements.

The literature suggests that task difficulty (for the operator) is a major component determining the use of combined head and eye movements during reading, and, as demonstrated in the Dunham (1997) study, for arithmetic processing as well. With respect 
to the results involving reading, young readers (6-10 year old), adults reading in a foreign language, adults who are slow readers, adults and children reading aloud, all demonstrate gaze shifts involving head movements while reading. We believe the common denominator for this effect is difficulty in abstracting information from the printed text. Whether this difficulty is restricted to cognitive difficulty or whether perceptual attributes produce similar effects has not been determined, or determined only to the extent of using longer than "normal" line lengths in the studies of Netchine and Lee. We suspect that any manipulation that increases difficulty of information abstraction leads to an increased likelihood of head movements. Whether the proportion of gaze shift attributable to head movements is affected by cognitive or perceptual difficulty has yet to be answered. Relating our results to issues of concern to the aviation community, might an increase in head movements relative to eye movements occur in Air Traffic Controllers during periods of higher than normal air traffic volume, and possibly provide a measure of the increasing task difficulty and possibly of incipient fatigue? As the complexity of cockpits increases, will the demand on the pilots both to fly the aircraft and intermittently obtain, assess, and act upon target information exceed the oculomotor and cognitive abilities, especially as the effects of sleep restriction and time on task fatigue accumulate? Understanding the relationship between head and eye movements, and the meaning of these movements with respect to the development of fatigue may lead to improvements in both the way we design our visual display systems and in the ways we train operators in their use.

One of the interesting aspects of Lee's (1999) study was the finding that two types of head movement could be identified, those associated with saccades, referred to as the "modulatory velocity component." The second component, labeled "constant velocity component" is associated with head movements in the direction of information abstraction and which occurs during fixations. He suggests that the latter component is associated with higher levels of information processing. Our observations of head movements in readers suggest that the "modulatory velocity component" associated with the saccade returning the eyes from the end to the beginning of a new line is the only one which is readily observable. We were puzzled by our inability to identify head movements to the right during reading of English text. It is quite possible that the "constant velocity component" identified by Lee is so slow that it cannot be visually detected. Since the written Korean language can be presented in the horizontal as well as vertical plane Lee studied both orientations and obtained similar results. He summarizes his results as follows:

"The pattern of head movement during reading was complex. Modulation of head velocity was not always coupled to a gaze saccade, and not all individual gaze saccades were accompanied by such modulation. Furthermore, occasionally no discernible modulation of head velocity was observed." P. 3767. We conclude with the suggestion that those concerned with evaluating gaze shifts under "normal" conditions, i.e. norestraint of the head, have to take into consideration both eye and head movements. The literature and our study suggest that task difficulty may be reflected in the use of head movements to shift gaze even when the required gaze shift is small. 
As may be obvious from our presentation, we have not converted EOG data to visual angle. We have not done so because visual angle calculated from calibration data, where head movements are minimal, would not be appropriate calibration values under conditions of head movement. Since we did not collect head movement data directly in this study, but had to infer it from the measures described, we believed it more reasonable to present the "raw" as compared to "converted" data.

This study explored the relationship between head movements and oculomotor measures for the purpose of advancing the discussion of operational application of visual system assessment. Incorporation of the results of studies such as this may lead to improvements in the methods of design of display systems and in methods of training operators required to use complex visual displays. Much work remains to be accomplished as visual systems become more challenged with increased information flow. With respect to the aviation community, the evaluation of the capabilities and limits of the oculomotor and visual cognition systems may be critical to saving lives of both crewmembers and passengers.

\section{Department of Defense Disclaimer}

Human volunteers participated in this study after giving their free and informed consent. Investigators adhered to AR 70-25 and USAMRDC Reg 70-50 on the use of volunteers in research. The opinions or assertions contained herein are the private views of the authors and are not to be construed as official or as reflecting the views of the Department of the Army or the Department of Defense.

\section{REFERENCES}

Baloh, R. W., Sills, A. W., Kumley, W. E., \& Honrubia, V. 1975. Quantitative measurement of saccade amplitude, duration, and velocity. Neurology, 25, 1065-1070.

Bartz, A. E. 1966. Eye and head movements in peripheral vision: Nature of compensatory eye movements. Science, 152, 1644-1655.

Dunham, D. N. 1997. Cognitive difficulty of a peripherally presented visual task affects head movements during gaze displacement. International Journal of Psychophysiology, 27, 171-182.

Fischer, F. P. 1924. Concerning head movements associated with visual information. Archive für Opthalmologie, 113, 394-416. (In German)

Fuller, J. H. 1992. Head movement propensity. Experimental Brain Research, 107, 156-164.

Goldring, J., Dorris, M. C., Corneil, B. D., Ballantyne, P., \& Munoz, D. P. 1996. Combined eyehead gaze shifts to visual and auditory targets in humans. Experimental Brain Research, 111, 68-78.

Hering, K. E. K. 1879. Raumsinn des Auges. Hermanns Handbuch der Physiologie. 3.Bd. Leipzig: F. C. W. Vogel.

Lee, C. 1999. Eye and head coordination in reading: roles of head movement and cognitive control. Vision Research, 39, 3761-3768.

McGregor, D. K. \& Stern, J. A. 1996. Time-on-task and blinks: Effects on saccade duration. Ergonomics, 39, 649-660.

McLean, W. E., \& Estrada, A. 2000. Feasibility of using the AN/PVS-14 Monocular Night Vision Device for Pilotage. (USAARL Technical Report No. 2000-18). Available on-line through library services at http://www.usaarl.army.mil/

Netchine, S., Greenbaum, C., \& Guihou, M.-C. 1984. Cephalic and ocular components of gaze displacement 
during oral and silent reading in children and adults. In A. G. Gale \& F. Johnson (Eds.), Theoretical and applied aspects of eye movement research (pp. 223-230). Amsterdam: Elsevier.

Netchine, S., Pugh, A. K., \& Guihou, M.-C. 1987. The organization of binocular vision in conjunction with head movements in French and English readers of 9 and 10 years. In J. K. O'Reagan \& F. LevySchoen (Eds.) Eye movements: From physiology to cognition (pp. 333-342). Amsterdam: Elsevier.

Netchine, S., Solomon, M., \& Guihou, M.-C. 1981. Composants oculairs et cephalique de l'organisation des deplacements du regards chez les jeunes lecteurs (Cephalic and ocular components of gaze displacement in children while reading). Psychologie Francaise, 26, 110-124.

Rash, C. E., McLean W. E., Mora, J. C., Ledford, M. H., Mozo, B. T., Licina, J. R., et al. 1998. Design issues for helmet-mounted display systems for rotary-wing aviation (USAARL Technical Report No. 98-32). Available on-line through library services at http://www.usaarl.army.mil/

Richards, J. E., \& Hunter, S. K. 1997. Peripheral stimulus localization by infants with eye and head movements during visual attention. Vision Research, 37, 3021-3035.

Ritzman. 1875. Über die Verwendung von Kopfbewegungen bei den gewöhnlichen Blickbewegungen. Archive für Opthalmologie, 21, 1. (In German)

Robinson, G. H., Koth, B. W., \& Ringenbach, J. P. 1976. Dynamics of the eye and head during an element of visual search. Ergonomics, 19, 691-709.

Sanders, A. F. 1970. Some aspects of the selective process in the functional visual field. Ergonomics, 13, 101-117.

Stern, J. A., \& Bynum, J. A. 1970. Analysis of visual search activity in skilled and novice helicopter pilots. Aerospace Medicine, 41, 300-305.

Tomlinson, R. D., \& Bahra, P. S. 1986a. Combined eye-head gaze shifts in the primate. I. Metrics. Journal of Neurophysiology, 56, 1542-1557.

Tomlinson, R. D., \& Bahra, P. S. 1986b. Combined eye-head gaze shifts in the primate. II. Interactions between saccades and the vestibuloocular reflex. Journal of Neurophysiology, 56, 1558-1570.

Troy, M. E., Chen, S. C., \& Stern, J. A. 1972. Computer analysis of eye movement patterns during visual search. Aerospace Medicine, 43, 390-394.

Zangemeister, W. H., Jones, A., \& Stark, L. 1981. Dynamics of head movement trajectories: Main sequence relationship. Experimental Neurology, 71, 76-91.

(Manuscript received November 2, 2004; Revision accepted January 30, 2005) 